JKAU: Eng. Sci., Vol. 20 No.1, pp: 3-28 (2009 A.D. / 1430 A.H.)

\title{
An ANN Based Fault Diagnosis System for Tapped HV/EHV Power Transmission Lines
}

\author{
E.A. Mohamed ${ }^{1}$, H.A. Talaat ${ }^{2}$ and E.A. Khamis ${ }^{3}$ \\ ${ }^{1,2}$ Elect. Power \& Machines Dept., Ain Shams Univ., Cairo, Egypt, \\ ${ }^{3}$ E E A, Nasr City, Cairo, Egypt, 1. Currently with Qassim University, \\ 2. Currently with King Saud University, KSA
}

Abstract. This paper presents a design for a fault diagnosis system (FDS) for tapped high/extra-high voltage (HV/EHV) power transmission lines (TL's). These tapped lines have two different protection zones. The proposed approach reduces the cost and the complexity of the FDS for these types of lines. The FDS, basically, utilizes fifteen artificial neural networks (ANN's) to reach its output diagnosis. The FDS basic objectives are mainly: 1. the detection of the system fault; 2 . the localization of the faulted zone; 3 . the classification of the fault type; and finally 4 . the identification of the faulted phase. This FDS is structured in a three hierarchical stages. In the first stage, a preprocessing unit to the input data is performed. An ANN, in the second stage, is designed in order to detect and zone localize the line faults. In the third stage, two zone diagnosis systems (ZDS) are designed. Each ZDS is dedicated to one zone and consists of seven parallel-cascaded ANN's. Four-parallel ANN's are designed in order to achieve the fault type classification. While, the other three cascaded ANN's are designed mainly for the selection of the faulted phase. A smoothing unit is also configured to smooth out the output response of the proposed FDS.

The proposed FDS is designed and evaluated using the local measurements of the three-phase voltage and current samples acquired at only one side. A sampling rate of 16 samples per cycle of the power frequency was taken. A data window of 4 samples was also utilized. These samples were generated using the EMTP simulation program, applied to the High-Dam/Cairo $500 \mathrm{kV}$ tapped TL. All possible shunt fault types were considered. The effect of fault location and fault incipience time were also included. Moreover, the effect of load and capacitor switchings on the FDS performance was investigated. Testing results have proved the capability as well as the effectiveness of the proposed FDS. 


\section{Introduction}

Protective relaying is one of the basic and necessary elements of an electric power system. The protective relaying role is to cause the prompt removal from service of any element, when it suffers a short circuit or when it starts to operate in an abnormal manner that may cause damage to the power system. In fact, in power systems, most of the faults occur on TL's. Faults MVA levels are usually high, and if they are not cleared rapidly they may cause system instability as well as damage and hazards to equipment and persons. Hence, the proper diagnosis or classification of TL faults is essential to the appropriate operation of power systems. Therefore, the fault type classification is a crucial protective relaying feature due to its significant effect on the operation enhancement of relaying scheme. The correct operation of major protective relays depends mainly on the fault classification feature ${ }^{[1-2]}$.

On the other hand, the faulted phase selection is as important as the fault detection. It would lead to an increase in the system stability and availability by allowing the healthy phases to operate using the single pole (or phase) tripping ${ }^{[3]}$. Single pole tripping has many benefits such as improving the transient stability and reliability of the power system, reducing the switching over-voltages and mitigating of the shaft torsional oscillations of large thermal units ${ }^{[3]}$. Figure 1 shows the essential structural modules of a modern protective relay, where the fault diagnosis modules are considered very important ones.

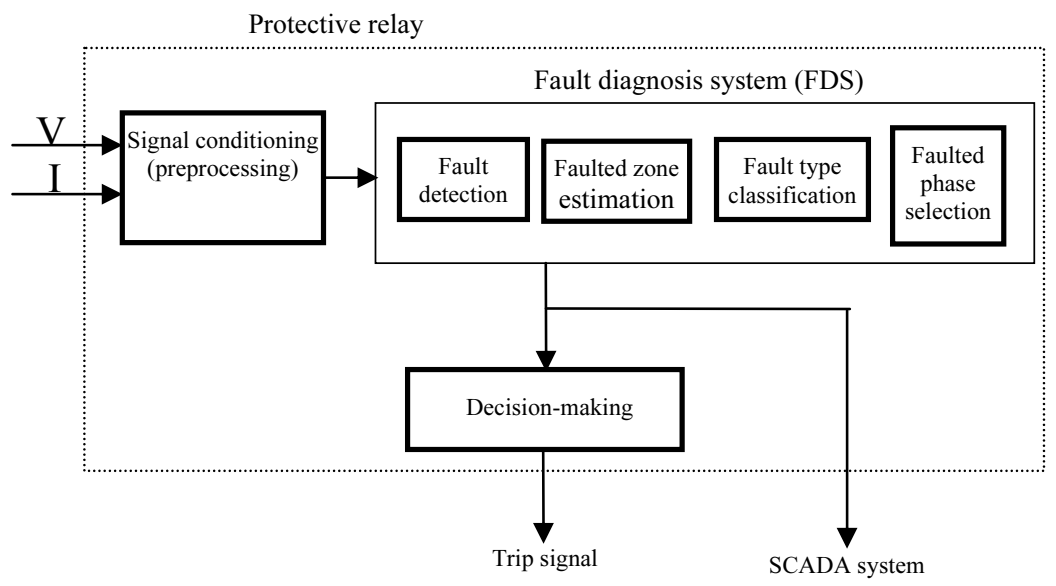

Fig. 1. Essential modules of a protective relay. 
Tapped TL's are those that are tapped usually through transformer bank primarily to supply loads. They are usually economical in their breakers requirements, but they need a complex relaying scheme for adequate protection and operation. These tapped TL's have two different protection zones, and faults must be detected and isolated in each zone individually. Simply, two different FDS's can be designed in order to achieve the standard requirements. Few research studies have been carried out on these types of TL's, due to the need of a very difficult protection scheme ${ }^{[1]}$. This study is trying to explore the design details of a proposed FDS, based on the application of ANN technology, as a part of the protection scheme required for these types of TL's.

The conventional analytical based classification approaches are expected to be affected by the system operating conditions. Also, a complete faulted phase selection can not be achieved through these approaches. Moreover, these approaches are also time consuming ${ }^{[1-4]}$. On the other hand, artificial intelligence techniques (expert systems, pattern recognition, ANN, and fuzzy logic) in general and ANN in particular provides a very interesting and valuable alternative ${ }^{[4]}$.

$\mathrm{ANN}^{[5-6]}$ can efficiently deal with most situations, which are not defined sufficiently for deterministic algorithms to execute. ANN can also accurately handle highly nonlinear tasks. Furthermore, ANN's are paralleled data-processing tools, capable of learning functional dependencies of data. Moreover, ANN's are robust with respect to incorrect or missing data. Protective relaying based ANN is not affected by a change in the system operating conditions. Also, ANN has fast computation rates, large input error tolerance and adaptive capabilities.

Many ANN architectures have been developed in different applications in the power industry ${ }^{[6-10]}$. On the other hand, many TL fault diagnosis or classification applications based on ANN technique have been introduced ${ }^{[11-15]}$. For example, an approach of two modules was developed $^{[11]}$ : a) The first module is designed for the fault type classification and phase selection, and b) The second module is designed for the classification of arcing and non-arcing faults. Samples of the three-phase voltages and currents with $1 \mathrm{kHz}$ sampling rate were used. In the first module, one ANN $(30 \times 20 \times 15 \times 11)$ of 30 input nodes, two hidden layers of 20 and 15 neurons, and 11 neurons in the output layer, was designed. A classification time in the range of 5-7 ms was achieved. In 
the second module, three ANN's $(20 \times 15 \times 10 \times 1)$ were designed and a classification time of 25-70 ms was reached.

Also, different hybrid TL fault diagnosis techniques have been implemented. In Ref. [16], a fuzzy/ANN hybrid classification approach is provided, a radial basis function (RBF) based ANN is employed for the TL classification system ${ }^{[17]}$, and a fuzzy/wavelet hybrid classification system for power system TL relaying is introduced ${ }^{[18]}$. The modular ANN is used for the design of the TL directional protection ${ }^{[19]}$. In Ref. [20\&21] a hybrid of fuzzy/ANN technique is implemented for the design of the classification scheme of power TL.

The purpose of this study is to design FDS using ANN capabilities, for the HV/EHV power tapped TL's. The conventional protection scheme for tapped TL's demands fewer requirements of breakers and higher complexity of relaying devices ${ }^{[1-3]}$. Simply, tapped TL's have two protection zones. Therefore, two different FDS's are required, in order to detect and isolate the fault in each zone individually. The proposed FDS is a one device scheme, designed to detect the fault, localize the faulted zone, classify the fault type and finally identify the faulted phase. Thus, the proposed FDS based relaying and protection scheme is less in cost and requirements. It consists basically of three hierarchical stages. It employs the modular ANN concept ${ }^{[19]}$. It has an output smoothing unit. Moreover, it is designed based on the simulation of tapped TL's using the EMTP program. Samples of the three phase voltages and currents at only the sending end are used as the input measurements. The High-Dam/Cairo $500 \mathrm{kV}$ tapped TL is the tested power TL in this study. All possible fault types are considered, the fault location and the fault incipience time are also implemented. Also, the effect of load and capacitor switchings on the FDS performance is evaluated. Testing results have proved the capability as well as the effectiveness of the proposed FDS.

\section{FDS Design Procedure}

An important module of the modern protective relay is the fault diagnosis one. The different functions required to be performed by the proposed FDS are: the fault detection, the faulted zone localization, the fault type classification and finally the faulted phase selection. Since the design procedure is a multi-task problem, it is preferable to decompose it into individual sub-problems, where each sub-problem is dedicated to one task. Using one ANN per task (modular $\mathrm{ANN}^{[19]}$ ) make it more 
powerful and will increase the learnability power of the ANN. Therefore, the proposed FDS consists of modular ANN, multi-ANN that are arranged to be working in parallel, which makes the proposed FDS more fast, efficient, robust, accurate and reliable.

The proposed FDS consists of 15 modular ANN's constructed in three hierarchical stages as illustrated in Fig. 2. The first stage is a preprocessing step involving data filtering, data conversion, and data normalization. In the second stage, one ANN (ANN\#1) designed to detect the faulted zone. It has a three output levels: low level $(<0.3)$ for normal condition, medium level ( 0.3 to 0.7 inclusive) for zone\#2 faults, and high level $(>0.7)$ for zone\#1 faults (nearest to the location of FDS). The third stage contains two parallel zone diagnosis systems (ZDS1 \& ZDS2). Each ZDS consists of 7 modular ANN's, designed to classify the fault type as well as select the faulted phase in its zone. ZDS1 is the fault diagnosis system for zone\#1. It consists of four parallel ANN's, each ANN of the first three, is cascaded with another ANN. The inputs to all ANN's are derived from the voltage and current samples acquired locally at the bus where the FDS is connected. The function of each ANN can be described as follows:

a. ANN11: The task of this ANN is to identify the phase to ground fault on zone\#1. It has two output levels: high level $(>0.5)$ for phase to ground fault and low level $(\leq 0.5)$ for normal condition and other fault types.

b. ANN12: This ANN is intended to classify the phase to phase fault on zone\#1. It has two output levels: high level $(>0.5)$ for phase to phase fault and low level $(\leq 0.5)$ for normal case and other fault types.

c. $A N N 13$ : This ANN is designed to recognize the double phase to ground fault at zone\#1. Its output is high level $(>0.5)$ for double phase to ground fault and low level $(\leq 0.5)$ for normal case and other fault types.

d. ANN14: This ANN is used to identify three phase to ground faults at zone\#1. Its output is high level $(>0.5)$ for three phase to ground fault and low level $(\leq 0.5)$ for else.

e. $A N N 111, A N N 121, A N N 131$ : These modular networks are designed to identify the faulted phase selection in case of single phase to ground, phase to phase, and double phase to ground faults respectively. The output of each ANN has three levels according to the faulted phases: low level $(<$ 0.3 ), medium level (0.3 to 0.7 inclusive) and high level (>0.7).

Similarly, ZDS2 is the zone fault diagnosis system for zone\#2. 


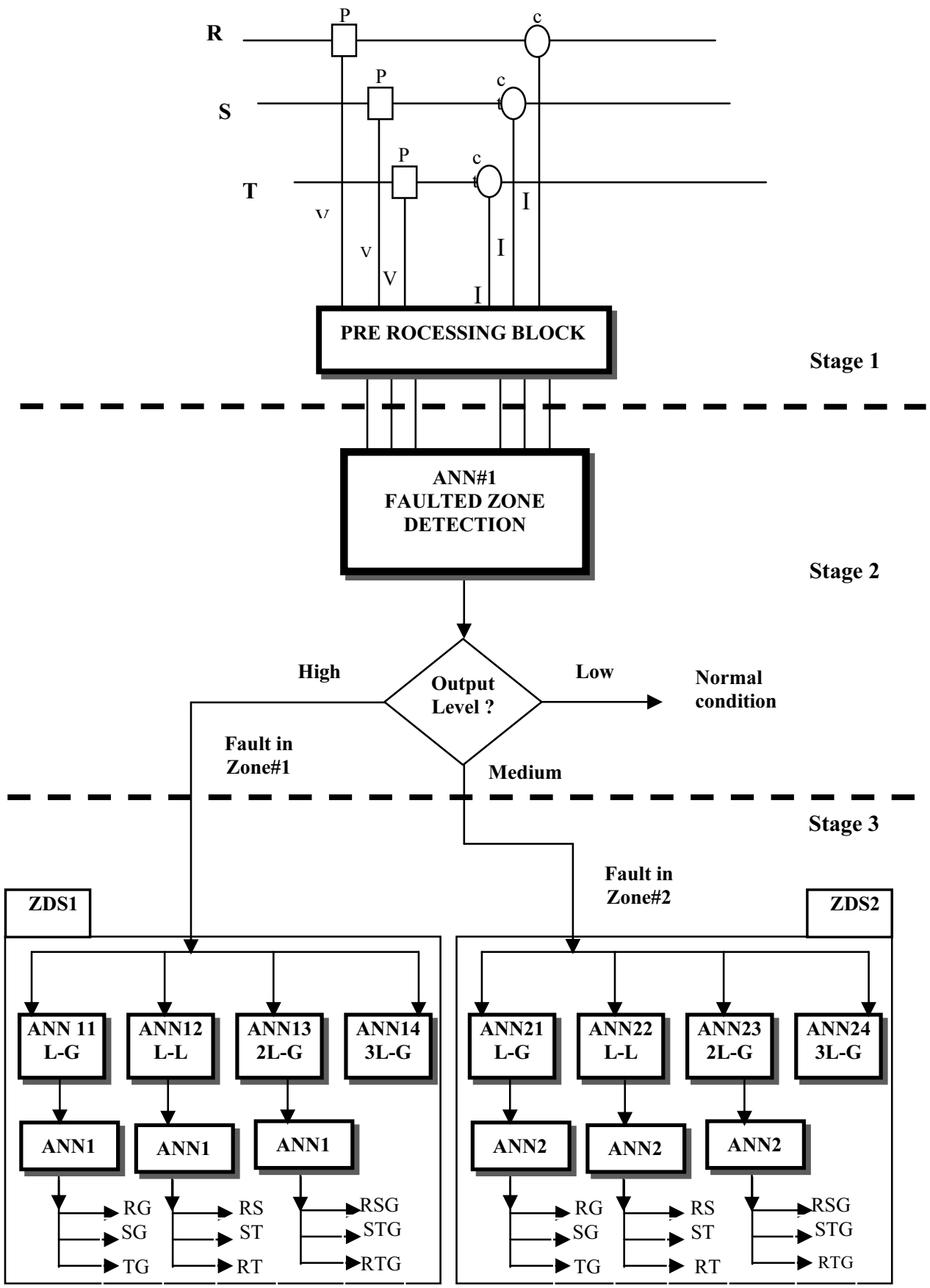

Fig. 2. Architecture of the suggested FDS. 
Moreover, Table 1 summarizes the role of design for each modular ANN, its output levels and its triggering mechanism. For example, ANN\#1 is designed, for the detection of faulted zone, with three output levels: high for faults on zone\#1, medium for faults on zone\#2, and low for normal conditions. Also, ANN11 (or ANN21) is designed, for the classification of fault type, with two output levels: high for L-G on zone\#1 (or zone\#2) and low for normal or other fault type conditions. These two networks are also triggered by ANN\#1 in case of fault condition. Moreover, ANN111 (or ANN211) is designed, for the selection of faulted phase, with three output levels: high for R-G fault on zone\#1 (or zone\#2), medium for S-G fault on zone\#1 (or zone\#2), and low for T-G fault on zone\#1 (or zone\#2). This network is triggered by ANN11 (or ANN21) in case of L-G fault. Similarly, the rest of similar networks are following a similar procedure. Three-phase TL labels are phase-R, phase$\mathrm{S}$, phase-T, and G-ground.

Table 1. ANN's role, output levels and its triggering network.

\begin{tabular}{|c|c|c|c|c|c|c|}
\hline \multirow{2}{*}{$\begin{array}{l}\text { Designed } \\
\text { ANN }\end{array}$} & \multirow{2}{*}{$\begin{array}{c}\text { Fault } \\
\text { type }\end{array}$} & \multirow{2}{*}{ Faulted zone } & \multirow{2}{*}{$\begin{array}{l}\text { Triggering } \\
\text { ANN }\end{array}$} & \multicolumn{3}{|c|}{ Output levels } \\
\hline & & & & High & Medium & Low \\
\hline ANN\#1 & $\begin{array}{c}\text { All } \\
\text { fault } \\
\text { types }\end{array}$ & $\begin{array}{l}\text { Zone\#1 or } \\
\text { zone\#2 }\end{array}$ & - & $\begin{array}{l}\text { Fault on } \\
\text { zone\#1 }\end{array}$ & $\begin{array}{c}\text { Fault on } \\
\text { zone\#2 }\end{array}$ & Normal \\
\hline \multicolumn{7}{|c|}{ Fault type classification } \\
\hline $\begin{array}{l}\text { ANN11 (or } \\
\text { ANN21) }\end{array}$ & L-G & $\begin{array}{c}\text { zone\#1 } \\
\text { (or zone\#2) }\end{array}$ & ANN\#1 & L-G & - & $\begin{array}{c}\text { Normal or other } \\
\text { fault types }\end{array}$ \\
\hline $\begin{array}{c}\text { ANN12 } \\
\text { (or ANN22) }\end{array}$ & L-L & $\begin{array}{c}\text { zone\#1 } \\
\text { (or zone\#2) }\end{array}$ & ANN\#1 & L-L & - & $\begin{array}{l}\text { Normal or other } \\
\text { fault types }\end{array}$ \\
\hline $\begin{array}{c}\text { ANN13 } \\
\text { (or ANN23) }\end{array}$ & $2 \mathrm{~L}-\mathrm{G}$ & $\begin{array}{c}\text { zone\#1 } \\
\text { (or zone\#2) }\end{array}$ & ANN\#1 & $2 \mathrm{~L}-\mathrm{G}$ & - & $\begin{array}{l}\text { Normal or other } \\
\text { fault types }\end{array}$ \\
\hline $\begin{array}{c}\text { ANN14 } \\
\text { (or ANN24) }\end{array}$ & $3 \mathrm{~L}-\mathrm{G}$ & $\begin{array}{c}\text { zone\#1 } \\
\text { (or zone\#2) }\end{array}$ & ANN\#1 & $3 \mathrm{~L}-\mathrm{G}$ & - & $\begin{array}{l}\text { Normal or other } \\
\text { fault types }\end{array}$ \\
\hline \multicolumn{7}{|c|}{ Faulted phase selection } \\
\hline $\begin{array}{c}\text { ANN111 } \\
\text { (or ANN211) }\end{array}$ & L-G & $\begin{array}{c}\text { zone\#1 } \\
\text { (or zone\#2) }\end{array}$ & $\begin{array}{c}\text { ANN11 } \\
\text { (or ANN21) }\end{array}$ & R-G & S-G & T-G \\
\hline $\begin{array}{c}\text { ANN121 } \\
\text { (or ANN221) }\end{array}$ & L-L & $\begin{array}{c}\text { zone\#1 } \\
\text { (or zone\#2) }\end{array}$ & $\begin{array}{c}\text { ANN12 } \\
\text { (or ANN22) }\end{array}$ & R-S & S-T & R-T \\
\hline $\begin{array}{c}\text { ANN131 } \\
\text { (or ANN231) }\end{array}$ & $2 \mathrm{~L}-\mathrm{G}$ & $\begin{array}{c}\text { zone\#1 } \\
\text { (or zone\#2) }\end{array}$ & $\begin{array}{c}\text { ANN13 } \\
\text { (or ANN23) }\end{array}$ & RS-G & ST-G & RT-G \\
\hline
\end{tabular}




\section{Numerical Application Results}

\subsection{System Under Study}

The present study is concerned with the protection of HighDam/Cairo $500 \mathrm{kV}$ tapped TL. The section from HD500 to NH500 is taken as zone\#1 and the length of this section is $235 \mathrm{~km}$. Zone\#2 is taken as the section from NH500 to AS500, of a length of $185 \mathrm{~km}$. This system is selected as an application example to design and evaluate the performance of the proposed FDS. Figure 3 shows the system arrangement and the location of the FDS as well. The power system data and operating conditions are given in Ref. [9] \& Appendix A.

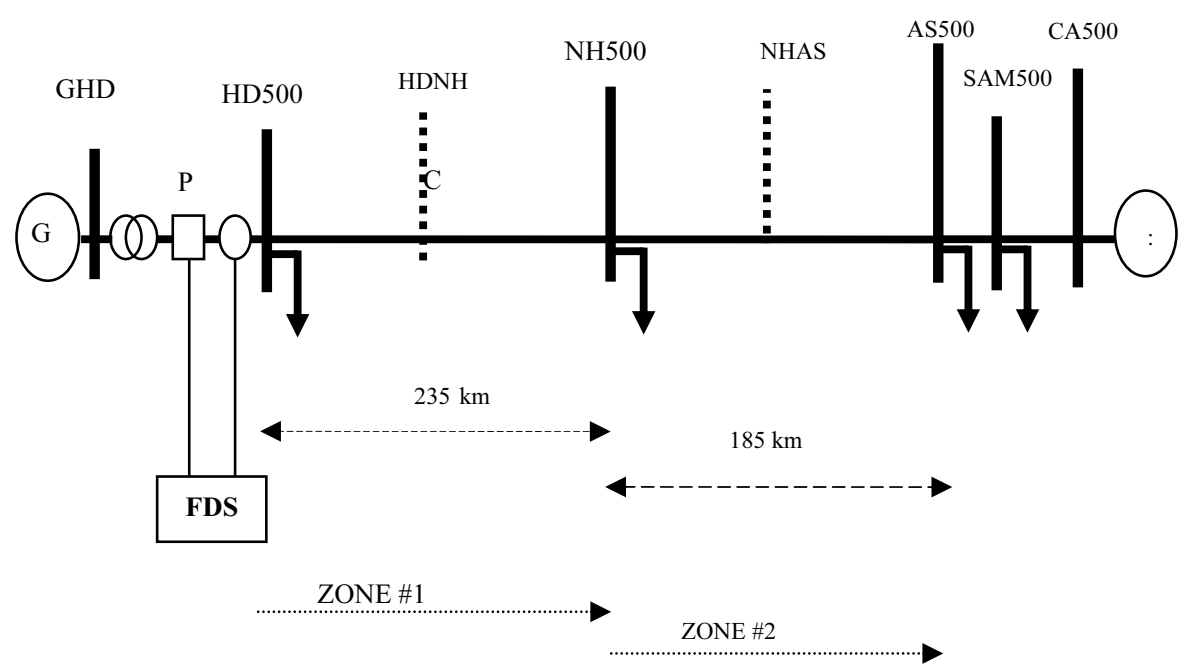

Fig. 3. System under study.

\subsection{Data Samples}

The simulation of the selected power system for this study is carried out using the electromagnetic transient program (EMTP $)^{[22]}$. The simulation is used to generate all fault patterns needed for the ANN design and evaluation processes. Different fault types (R-G, S-G, T-G, R-S, S-T, R-T, RS-G, ST-G, RT-G, RST-G) are considered. For each fault type, fault locations are selected at each bus and on midpoints, also the fault incipience times are taken at zero crossing and the peak.

\section{Number of fault conditions $=$}

10 fault types $\times 5$ fault locations $\times 2$ fault incipience times 
Therefore, there are 100 case studies. Each case study contains threecycles during the fault and three-cycles after the fault clearance. In addition, one-cycle before the fault is taken into consideration for only two case studies, one for zone\#1 and the other for zone\#2. Accordingly, the number of samples is $(2$ cases $\times 7$ cycles $\times 16$ samples/cycle +98 cases $\times 6$ cycles $\times 16$ samples/cycle $=9632$ samples $)$. The generated samples then divided into two sets; the design (training) set which is composed of 6752 samples (70 case studies), and the evaluation (testing) set is composed of 2880 samples (30 different case studies).

\subsection{ANN Design Procedure}

EMTP generated case studies are then loaded into the MATLAB software. The voltages and currents are normalized and reshaped in the form of group of patterns. Each pattern is composed of a predefined number of consecutive samples. Different data windows are examined (one, two, or four samples). ANN Toolbox of the MATLAB software ${ }^{[23]}$ is used to design each modular ANN, as assigned in the proposed design scheme of the FDS. Figure 4 shows the different steps required for the design procedure for each modular ANN (for more details see Ref. [9]).

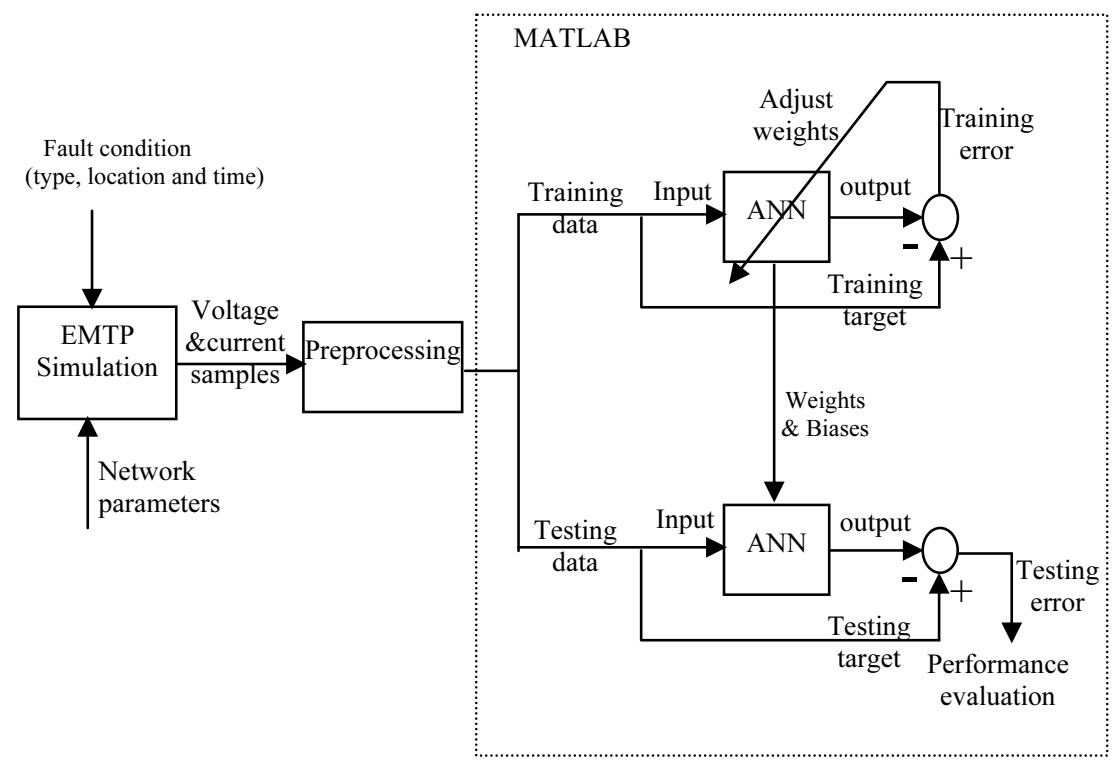

Fig 4. Steps of ANN design. 


\subsection{ANN Adopted Structure}

There is no definite way for pre-determining the optimum network structure without testing different configurations. The three layer feedforward ANN was found satisfactory for pattern classification problems $^{[11-13]}$. Therefore, it is chosen for this application. The tansigmoid and the log-sigmoid activation functions are differentiable as well as monotonic functions; therefore, they are employed as the network activation functions. Thus, the network structure will be one hidden layer with the tan-sigmoid neurons followed by an output layer with the logsigmoid neurons.

The number of input variables and number of neurons in the hidden layer are decided using the experimentation process that involves different network configurations. The process will be terminated when a suitable network structure is achieving a satisfactory performance. Each ANN is designed and evaluated using the three-phase voltage and current samples with the sampling rate of 16 samples per cycle. Different data windows (number of samples per pattern), one sample, two samples, oneRMS sample, two-RMS samples and four consecutive samples are examined. The best FDS performance was obtained using a data window of four consecutive samples. Based on the above mentioned, the appropriate structure for each modular ANN used in this application is: 24 input nodes, one hidden layer with 24 tan-sigmoid neurons, an output layer with one log-sigmoid neuron. Figure 5 shows the adopted structure. Where W1 \& W2 are the first \& second hidden layer weight matrices, respectively. While B1 \& B2 are the bias vectors for the first \& second hidden layer, respectively (K: a sample index).

\section{FDS Performance Evaluation}

\subsection{Localization of Faulted Zone and Classification of Fault Type}

Completing the design procedure, the FDS is then evaluated at different fault conditions, i.e., at different fault locations, different fault types and different fault incipience time (i.e., 30 case studies). These cases are different from those used for the design procedure. 


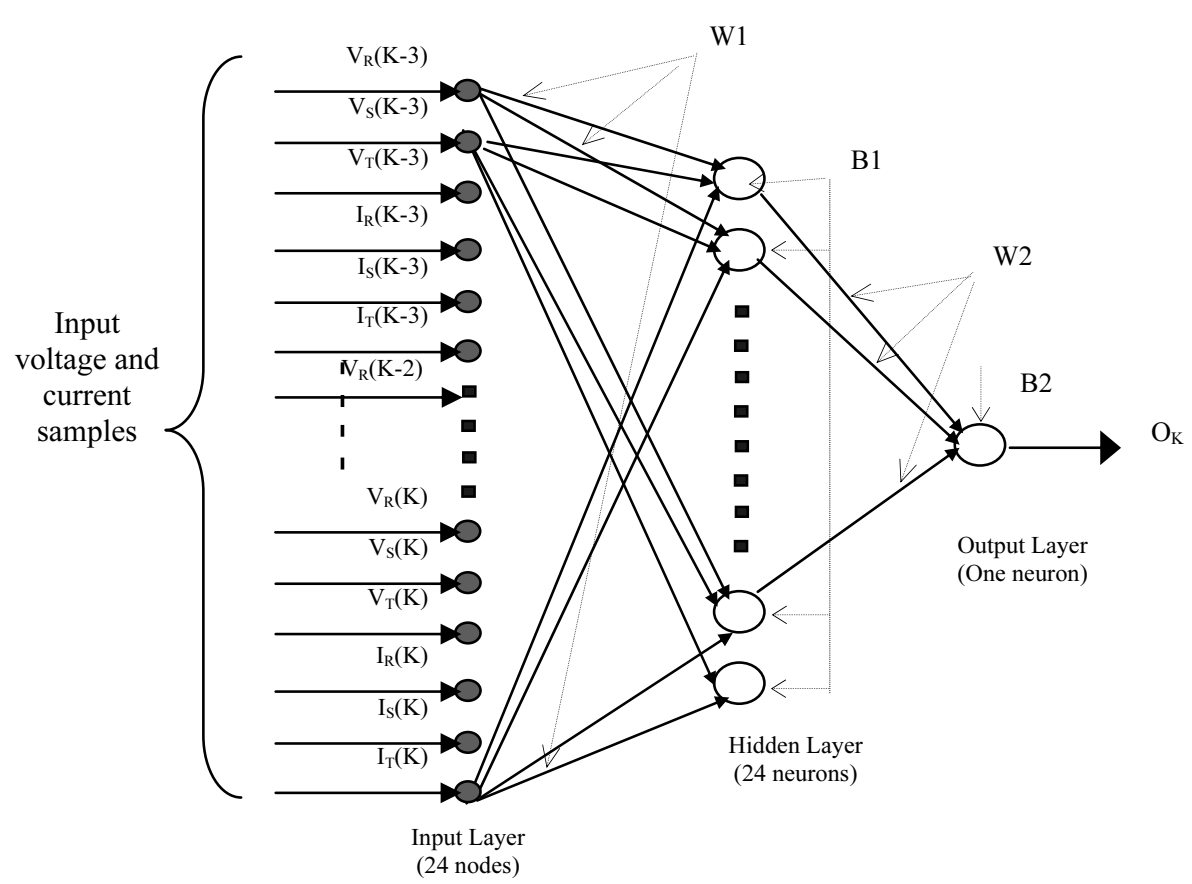

Fig. 5. Adopted ANN structure.

\subsubsection{Phase to Ground Faults}

Figure 6 shows the three-phase voltages and currents, due to S-G fault at HD500, ANN\#1-output and the outputs of other ANN's (ANN11, ANN12, ANN13, ANN14), which are responsible for classifying the fault type at zone\#1. As shown in the figure, when the fault is triggered the voltage of phase $\mathrm{S}$ collapses and its corresponding phase current increases. The fault is then cleared after 3 cycles. ANN\#1-output flags at a high level $\approx 0.9$ (as expected for a fault on zone\#1) during the fault duration and falls to a low level $\approx 0.1$ when the fault is cleared after a duration of other 3 cycles. This proves that the network has localized the faulted zone accurately. The outputs of other ANN's are at low level $\approx$ 0.1 except ANN11, which is designed for detecting L-G faults. The output of ANN11 is almost at high level $\approx 0.9$ during the fault duration and falls to a low level $\approx 0.1$ when the fault is cleared. Therefore, the performance of the FDS in this case is accurate. It must be noted here that all ANN outputs are smoothed out using the output smoothing unit 
which performs an average over each 4 samples. Therefore, each ANN output is 24 response points (pattern index).
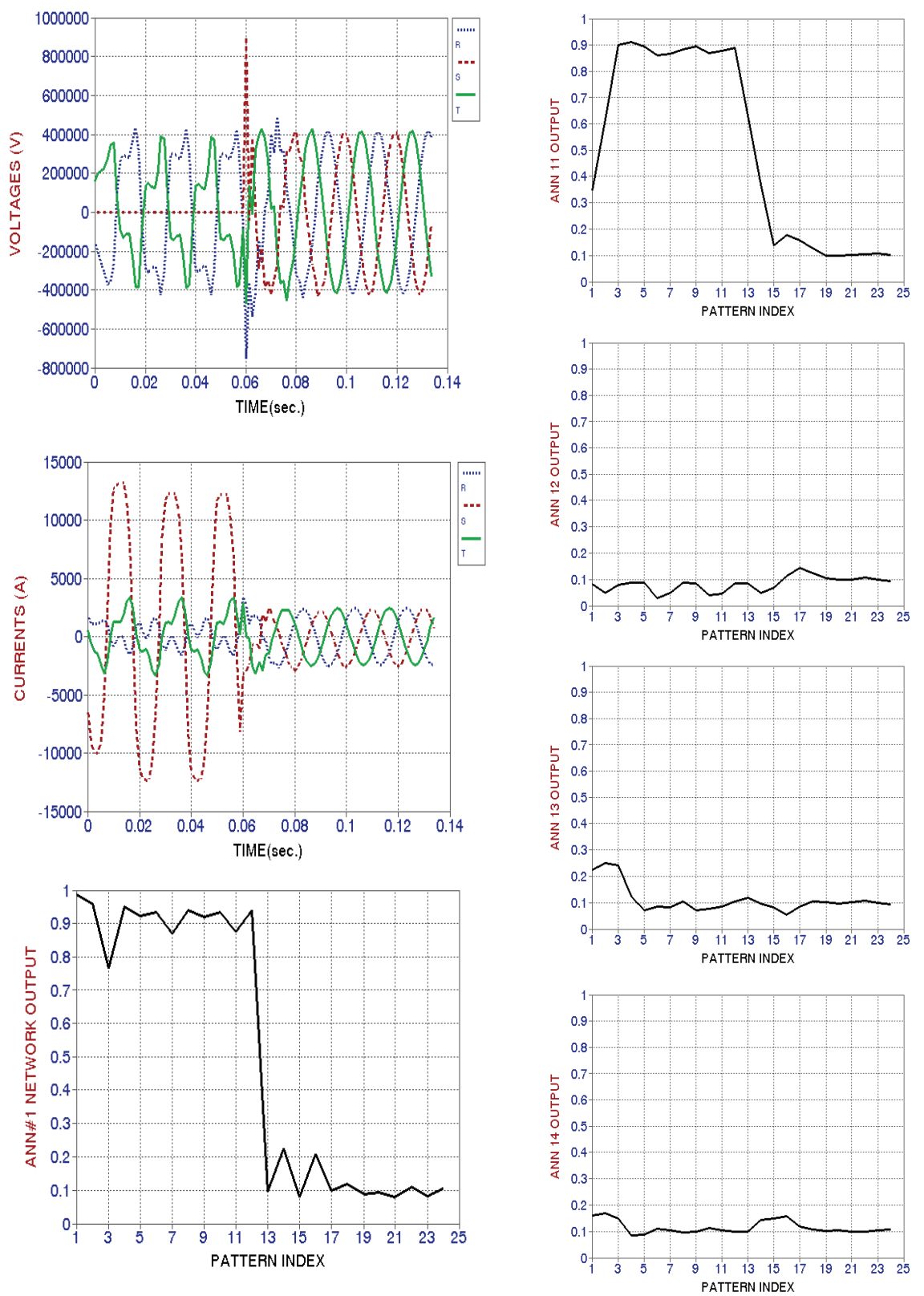

Fig. 6. Three phase voltages and currents at FDS and corresponding ANN outputs due to S-G fault at HD500. 
Figure 7 shows the voltage waveform and the increasing current of phase S due to S-G fault at AS500. ANN\#1-output is at a medium level $\approx$ 0.5 (as expected for a fault on zone\#2) during the fault duration then falls to a low level $\approx 0.1$ when the fault is cleared. This proves that the network detection capability of the faulted zone is accurate. The output of ANN21, designed for classifying L-G faults on zone\#2, is almost at a high level $\approx 0.9$ during the fault and at a low level $\approx 0.1$ after clearing the fault. The outputs of the other three ANN's (ANN22, ANN23 \& ANN24) are at a low level $\approx 0.1$ all over the fault and fault cleared periods, as it is expected.
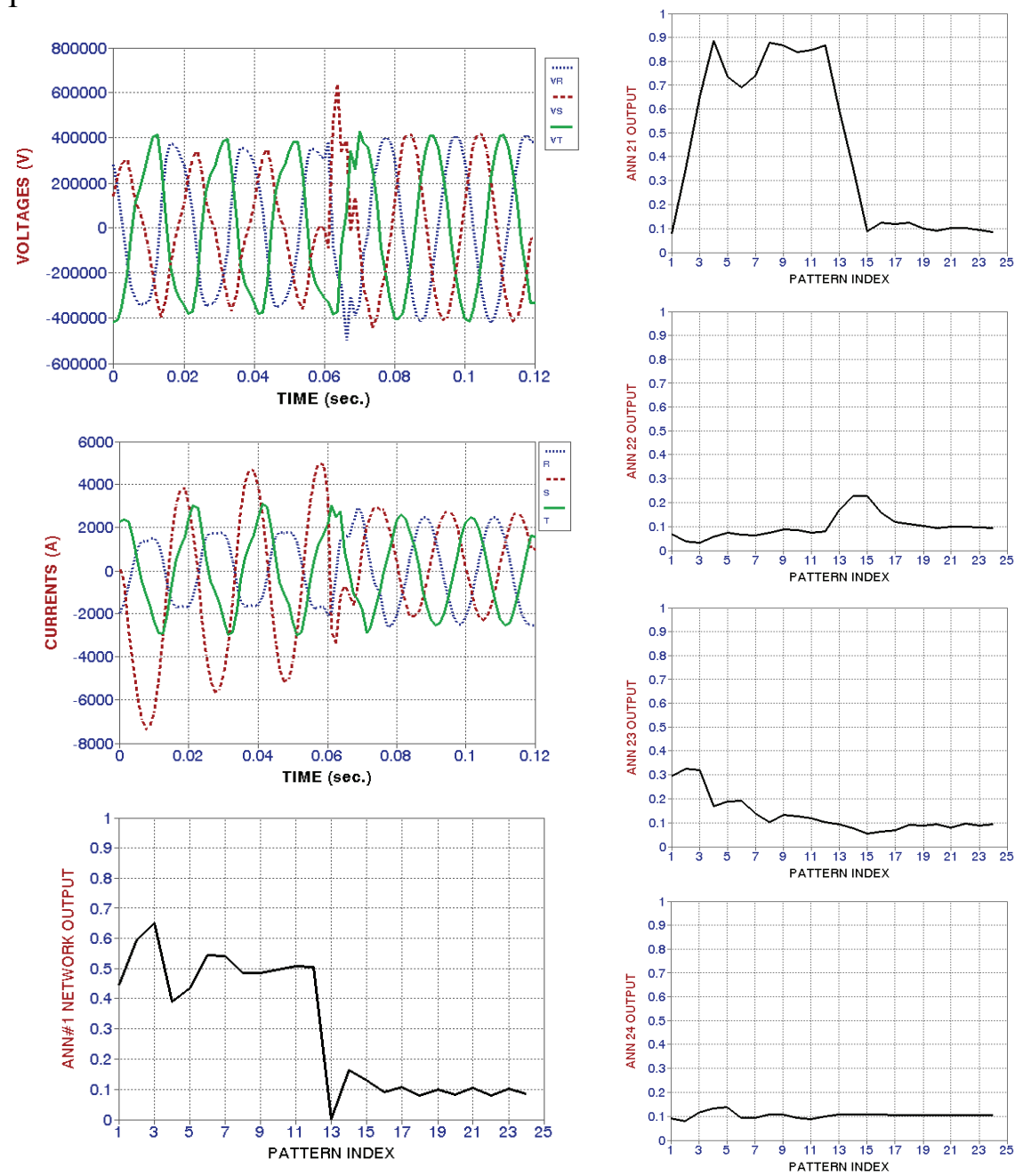

Fig. 7. Three phase voltages and currents at FDS and corresponding ANN outputs due to S-G fault at AS500. 


\subsubsection{Phase to Phase Faults}

Figure 8 shows the output response of ANN\#1 at almost a high level $\approx 0.9$ (as expected for fault on zone \#1) during the S-T fault at NH500, then falls to a low level $\approx 0.1$ after clearing the fault. The output response of ANN12 is at a high $\approx 0.9$ and low $\approx 0.1$ levels during and after the fault, respectively. The outputs of the other three ANN's are all at a low level $\approx 0.1$ allover the period, as it should be.
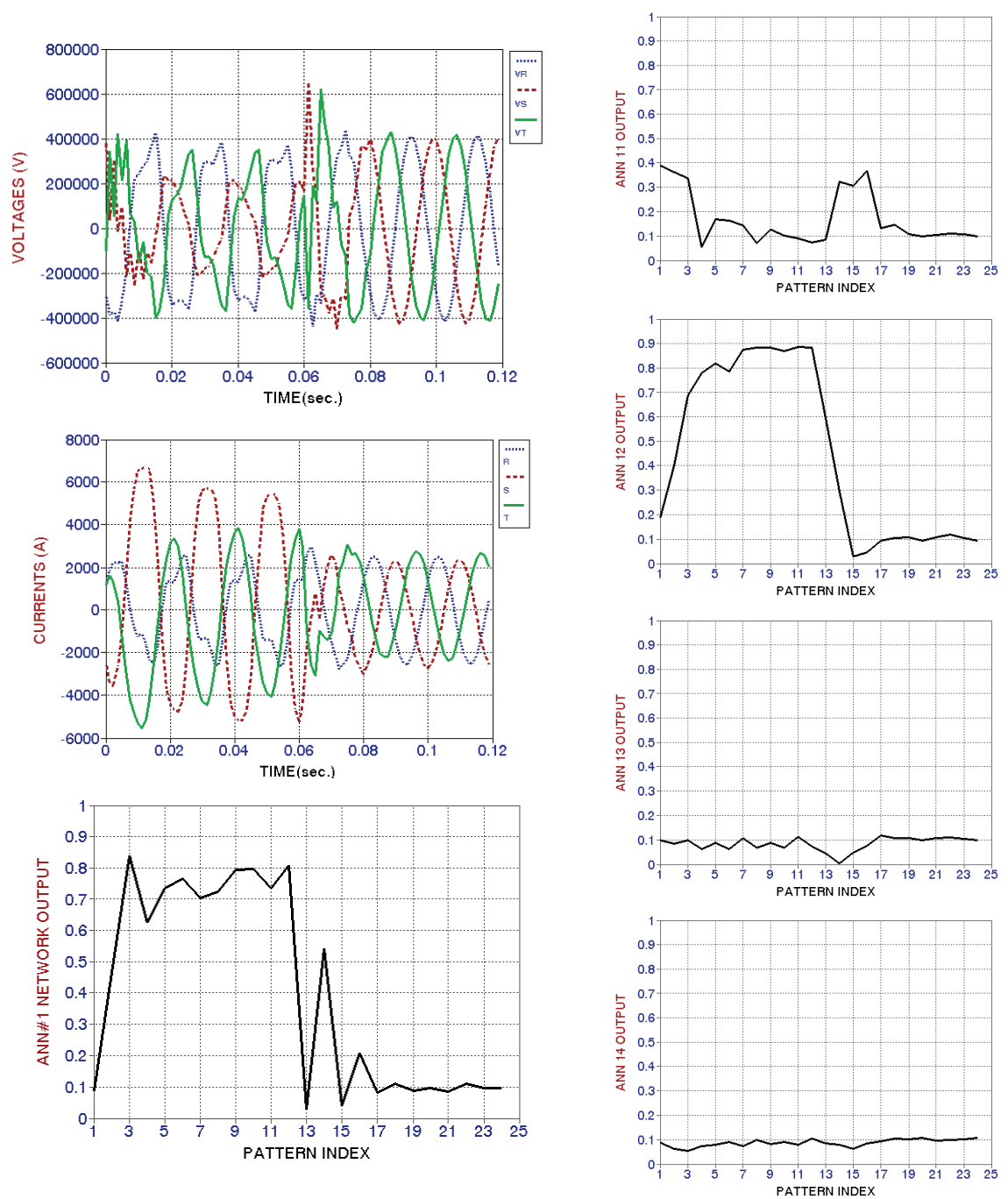

Fig. 8. Three phase voltages and currents at FDS and corresponding ANN outputs due to ST fault at NH500. 
Figure 9 shows the output response, due to S-T fault at NHAS, of ANN\#1 as well as the outputs of the other ANN's (ANN21, ANN22, ANN23\& ANN24), which are responsible for classifying the fault type on zone\#2. ANN\#1 output is at a medium level $\approx 0.5$ during fault then falls to a low level $\approx 0.1$ after the fault is cleared. The output of other ANN's are all at a low level $\approx 0.1$ except ANN22 designed to detect L-L faults (at a high level $\approx 0.9$ during fault).
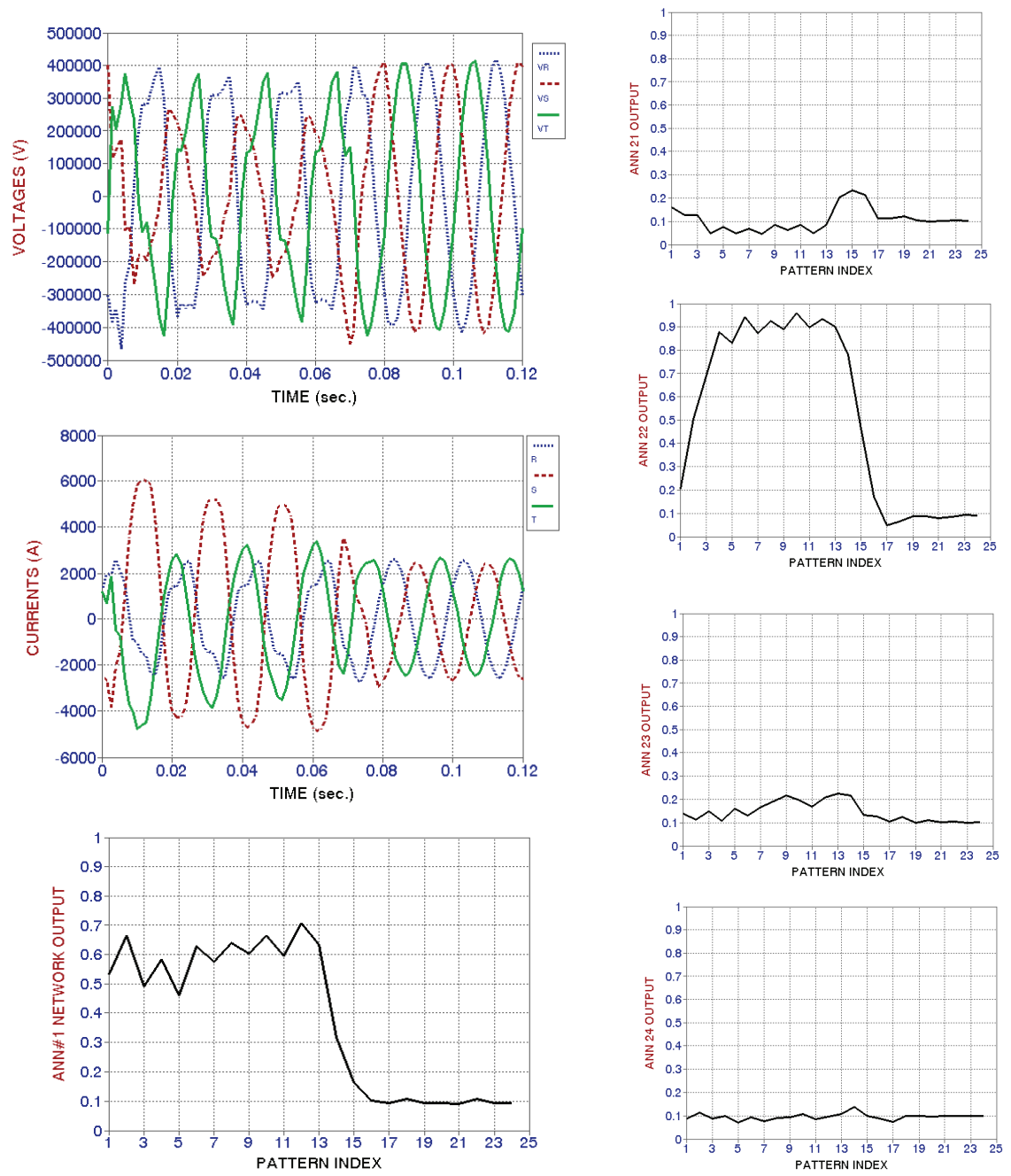

Fig. 9. Three phase voltages and currents at FDS and corresponding ANN outputs due to S-T fault at NHAS. 


\subsubsection{Double Phase to Ground Faults}

Figure 10 shows the FDS response due to RS-G fault at HDNH, where ANN\#1-output responds a high level $\approx 0.9$ during fault and then falls to a low level $\approx 0.1$ after clearing fault. ANN13 response is at high level $\approx 0.9$ during fault and low level $\approx 0.1$ after clearing the fault, and other ANN's outputs are at low levels $\approx 0.1$ allover the period.
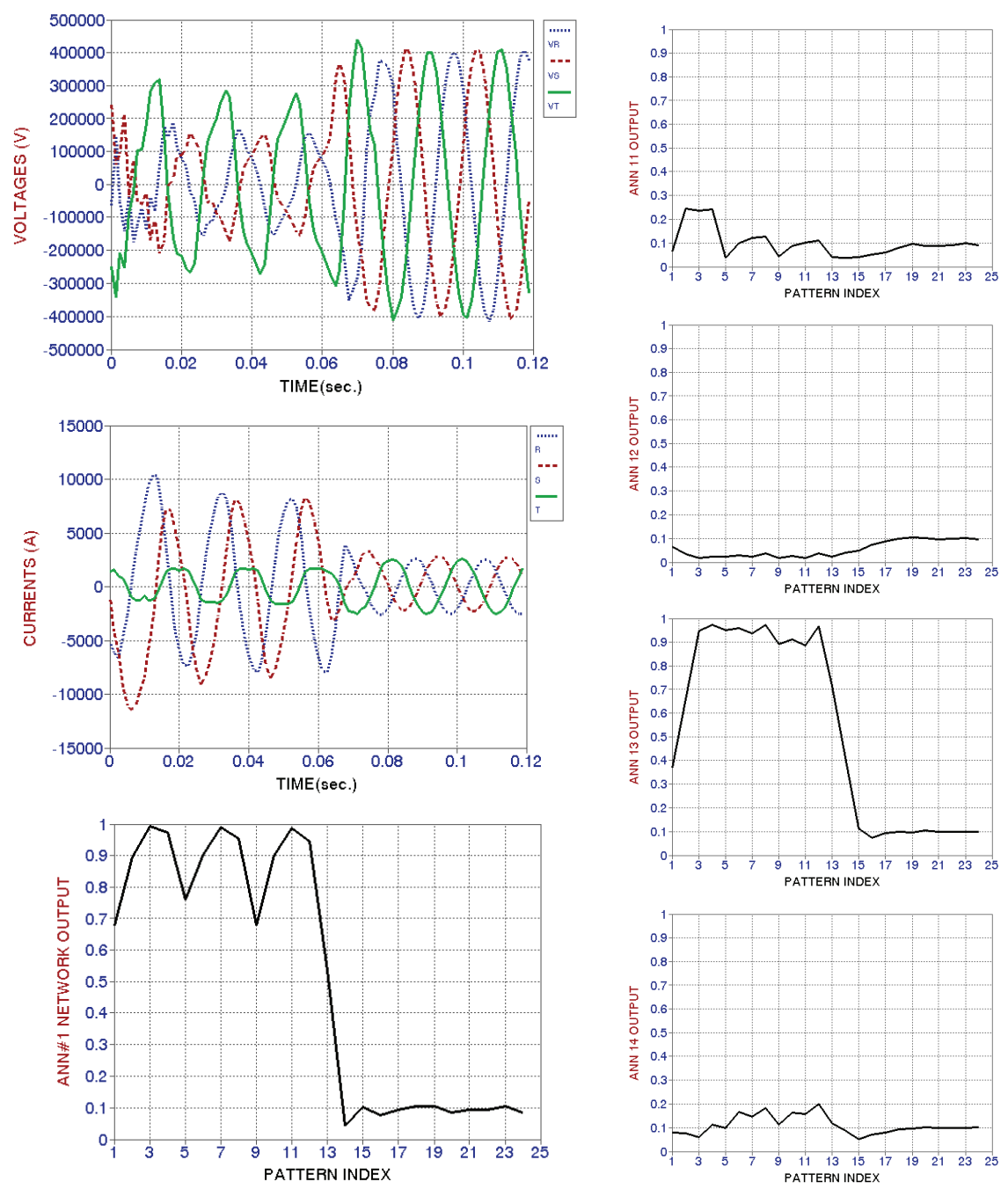

Fig. 10. Three phase voltages and currents at FDS and corresponding ANN outputs due to RS-G fault at HDNH. 
Similarly, Fig. 11 shows the FDS response due to ST-G fault at NHAS. The output of ANN\#1 is almost at a medium level $\approx 0.5$ during fault and falls to a low level $\approx 0.1$ after the fault is cleared. The outputs of other ANN's are also at low level $\approx 0.1$ except ANN23, which is designed to detect $2 \mathrm{~L}-\mathrm{G}$ faults, is at high level $\approx 0.9$ during fault.
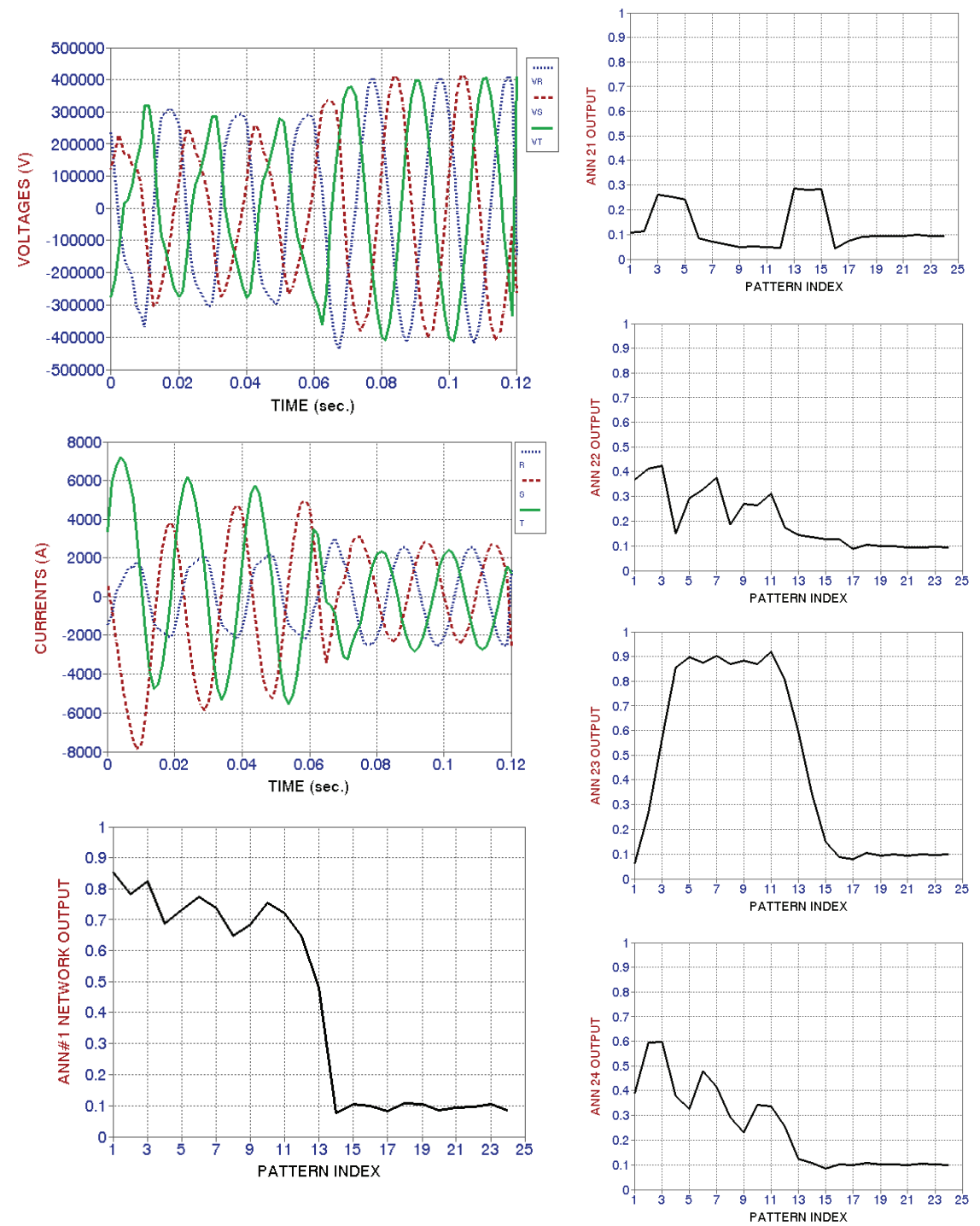

Fig. 11. Three phase voltages and currents at FDS and corresponding ANN outputs due to ST-G fault at NHAS. 


\subsubsection{Three Phase to Ground Faults}

Figure 12 presents the three-phase voltages and currents due to $3 \mathrm{~L}-\mathrm{G}$ fault at HDNH, the output of ANN\#1 and the outputs of other ANN's. ANN\#1 output is at a high level $\approx 0.9$ during fault and falls to a low level $\approx 0.1$ after clearing the fault. Other ANN's output are all at low level $\approx$ 0.1 except ANN14, designed to detect $3 \mathrm{~L}-\mathrm{G}$ faults, is at high level $\approx 0.9$ during fault.
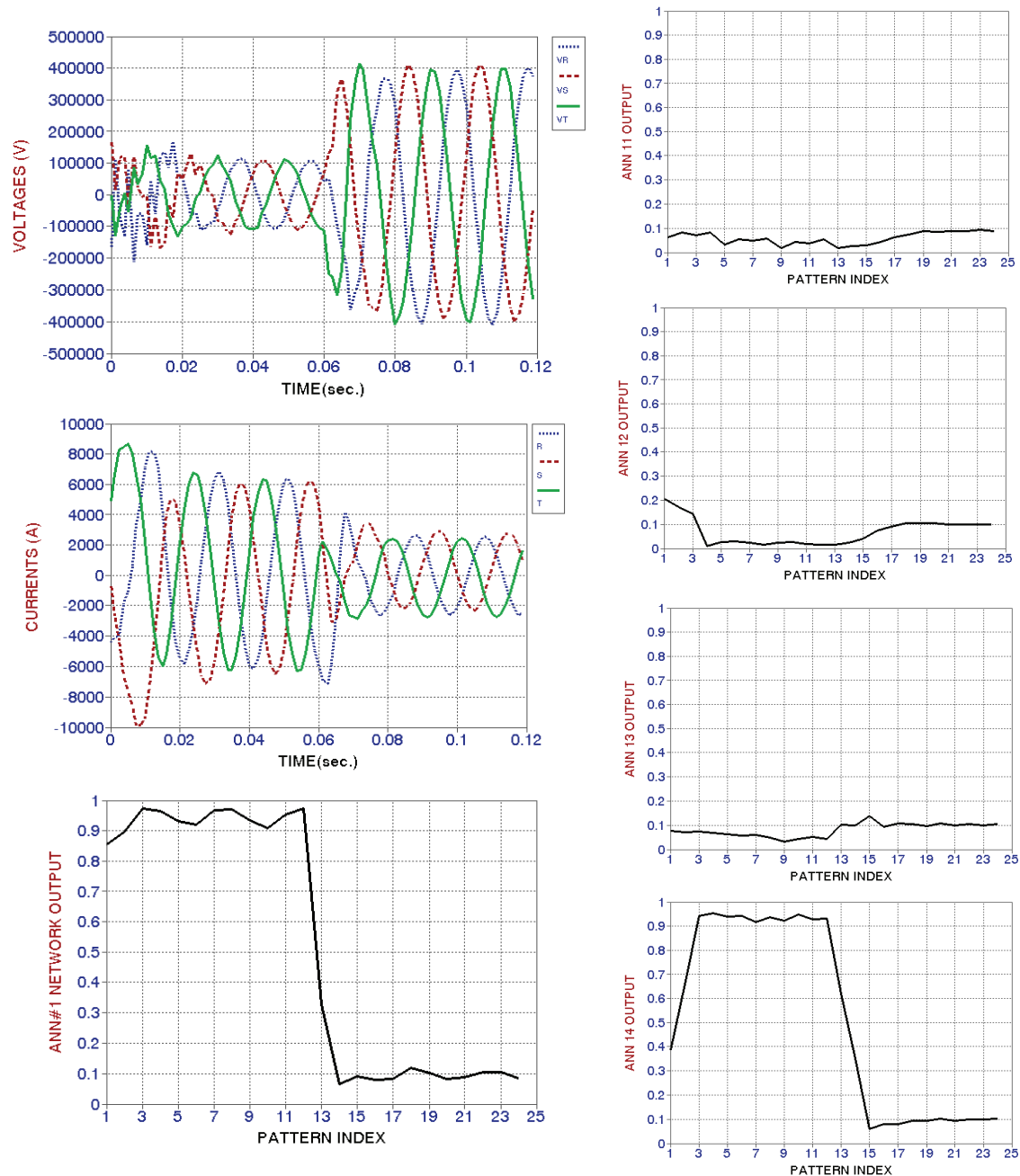

Fig. 12. Three phase voltages and currents at FDS and corresponding ANN output due to 3L-G fault at HDNH. 
Similarly, Fig. 13 shows, due to 3L-G fault at AS500, the output of ANN\#1 and the outputs of other ANN's (responsible for classifying the fault type at zone\#2). ANN\#1 output is at medium level $\approx 0.5$ during fault and falls to a low level $\approx 0.1$ after clearing the fault. Other ANN's output are all at low level $\approx 0.1$ except ANN24, designed to detect 3L-G faults, is almost at high level $\approx 0.9$ during fault.
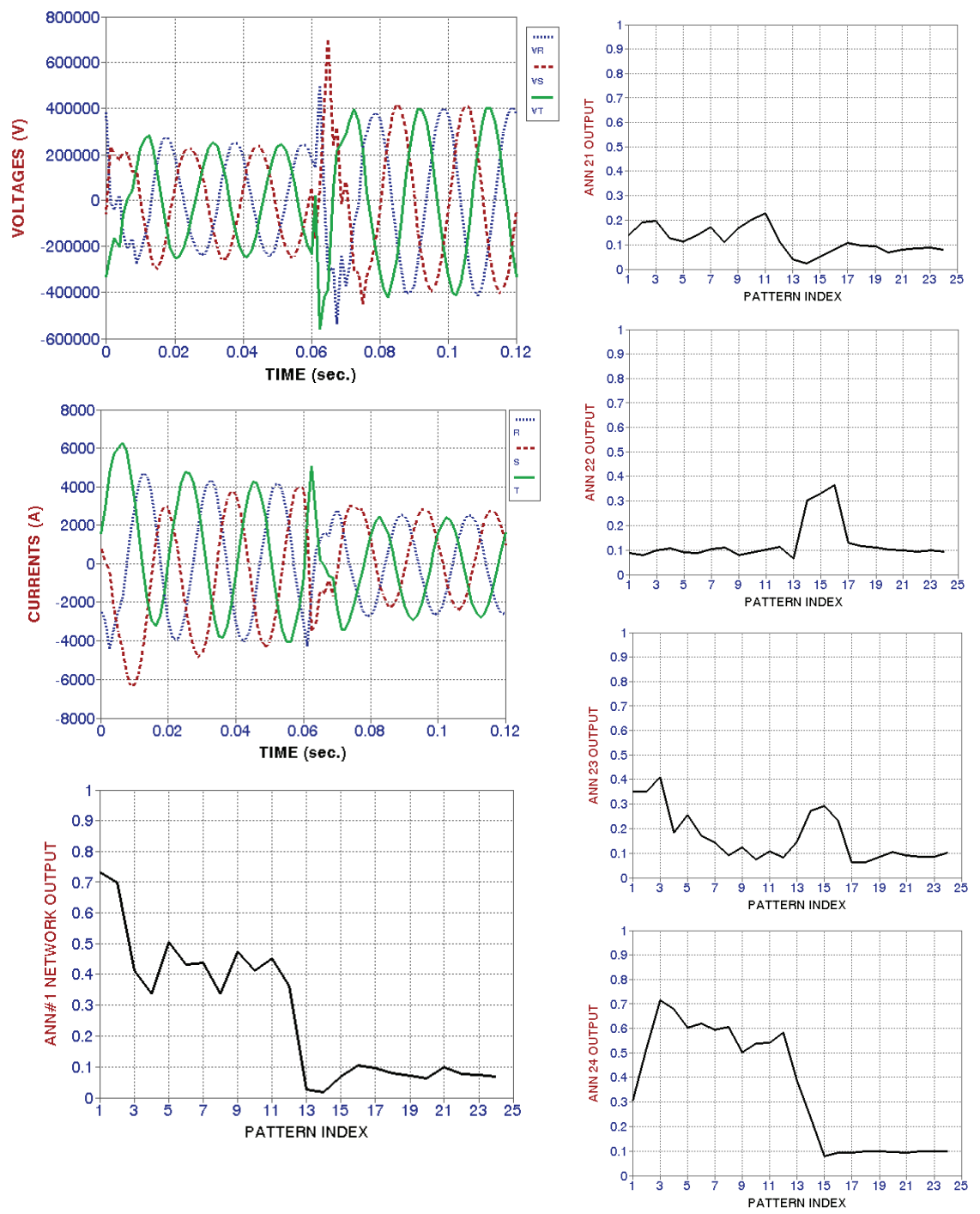

Fig. 13. Three phase voltages and currents at FDS and corresponding ANN outputs due to 3L-G fault at AS500. 


\subsection{Phase Selection}

The testing results of the faulted phase selection on zone\#1 are summarized in Table 2. From these results, it can be seen that for each ANN of the three specified ANN's per zone (e.g., ANN111, ANN121, and ANN131 for zone\#1), the response during fault (12 data points) matches well with the corresponding target value. This means that the ANN response is fairly satisfactory regarding the phase selection for each fault. Therefore, it can be stated that the FDS phase selection capability is very promising.

Table 2. Results of faulted phase selection on zone\#1.

\begin{tabular}{|c|c|c|c|c|c|c|c|c|c|}
\hline $\begin{array}{c}\text { ANN } \\
\text { no. }\end{array}$ & \multicolumn{3}{|c|}{ ANN 111 } & \multicolumn{3}{c|}{ ANN 121 } & \multicolumn{3}{c|}{ ANN 131 } \\
\hline $\begin{array}{c}\text { Fault } \\
\text { location }\end{array}$ & HDNH & HDNH & HDNH & HDNH & HD500 & NH500 & HDNH & HD500 & NH500 \\
\hline Phase & SG & RG & TG & RS & ST & RT & RSG & RTG & STG \\
\hline Target & 0.5 & 0.1 & 0.9 & 0.1 & 0.5 & 0.9 & 0.1 & 0.9 & 0.5 \\
\hline \multirow{7}{*}{\begin{tabular}{c} 
ANN \\
\cline { 2 - 12 }
\end{tabular}} & 0.38060 & 0.09675 & 0.90307 & 0.1164 & 0.5703 & 0.8728 & 0.1352 & 0.9341 & 0.2825 \\
\cline { 2 - 12 } & 0.45535 & 0.06084 & 0.90559 & 0.0954 & 0.4130 & 0.7703 & 0.1038 & 0.6434 & 0.5402 \\
\cline { 2 - 11 } & 0.49774 & 0.08480 & 0.90907 & 0.0772 & 0.4422 & 0.9128 & 0.0900 & 0.8993 & 0.5793 \\
\cline { 2 - 11 } & 0.53031 & 0.05153 & 0.92546 & 0.0768 & 0.5476 & 0.8107 & 0.0615 & 0.8949 & 0.5546 \\
\cline { 2 - 11 } & 0.49129 & 0.07699 & 0.90534 & 0.0990 & 0.5230 & 0.9289 & 0.0849 & 0.9258 & 0.4905 \\
\cline { 2 - 11 } & 0.48467 & 0.05311 & 0.92398 & 0.0916 & 0.4976 & 0.8515 & 0.0881 & 0.6822 & 0.5044 \\
\cline { 2 - 11 } & 0.49370 & 0.05962 & 0.91464 & 0.0845 & 0.5038 & 0.7934 & 0.1009 & 0.8290 & 0.5217 \\
\cline { 2 - 10 } & 0.46369 & 0.05372 & 0.92335 & 0.0942 & 0.4985 & 0.8448 & 0.0896 & 0.6964 & 0.5036 \\
\cline { 2 - 10 } & 0.47267 & 0.08478 & 0.90542 & 0.0976 & 0.4965 & 0.9222 & 0.1053 & 0.8987 & 0.4865 \\
\cline { 2 - 10 } & 0.48868 & 0.06106 & 0.91042 & 0.0887 & 0.4967 & 0.7909 & 0.1072 & 0.8078 & 0.509 \\
\hline
\end{tabular}

\subsection{Effect of Load and Capacitor Switching}

Load or capacitance switching may generate transients in the power system, which can be misclassified as fault case. Hence, the performance of the proposed FDS is evaluated using these two cases of switchings. Figure 14 shows the three-phase voltages and currents and the corresponding output response of ANN\#1, due to the switching of a capacitor at NH500 bus. On the other hand, Figure 15 shows the three- 
phase voltages and currents and the associated output response for ANN\#1, due to the switching of an inductive load at NH500 bus. As shown from these figures, the voltage and current waveforms are affected but the output of ANN\#1 is still low allover the period indicating a normal condition. Therefore, the proposed FDS is insensitive to the load and capacitor switchings.
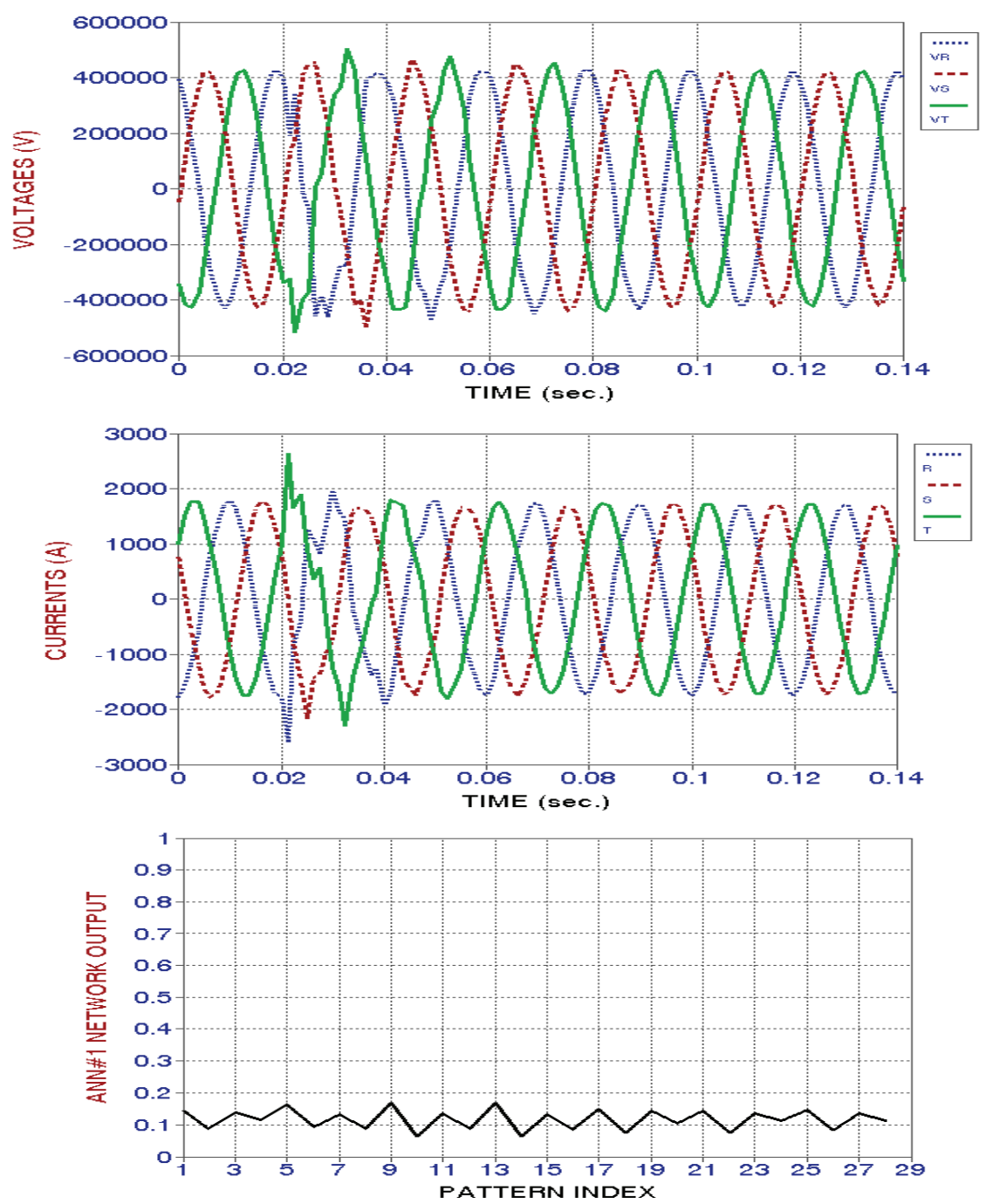

Fig. 14. Three phase voltages and currents at FDS and corresponding ANN outputs due to capacitor switching at $\mathbf{N H 5 0 0 .}$ 

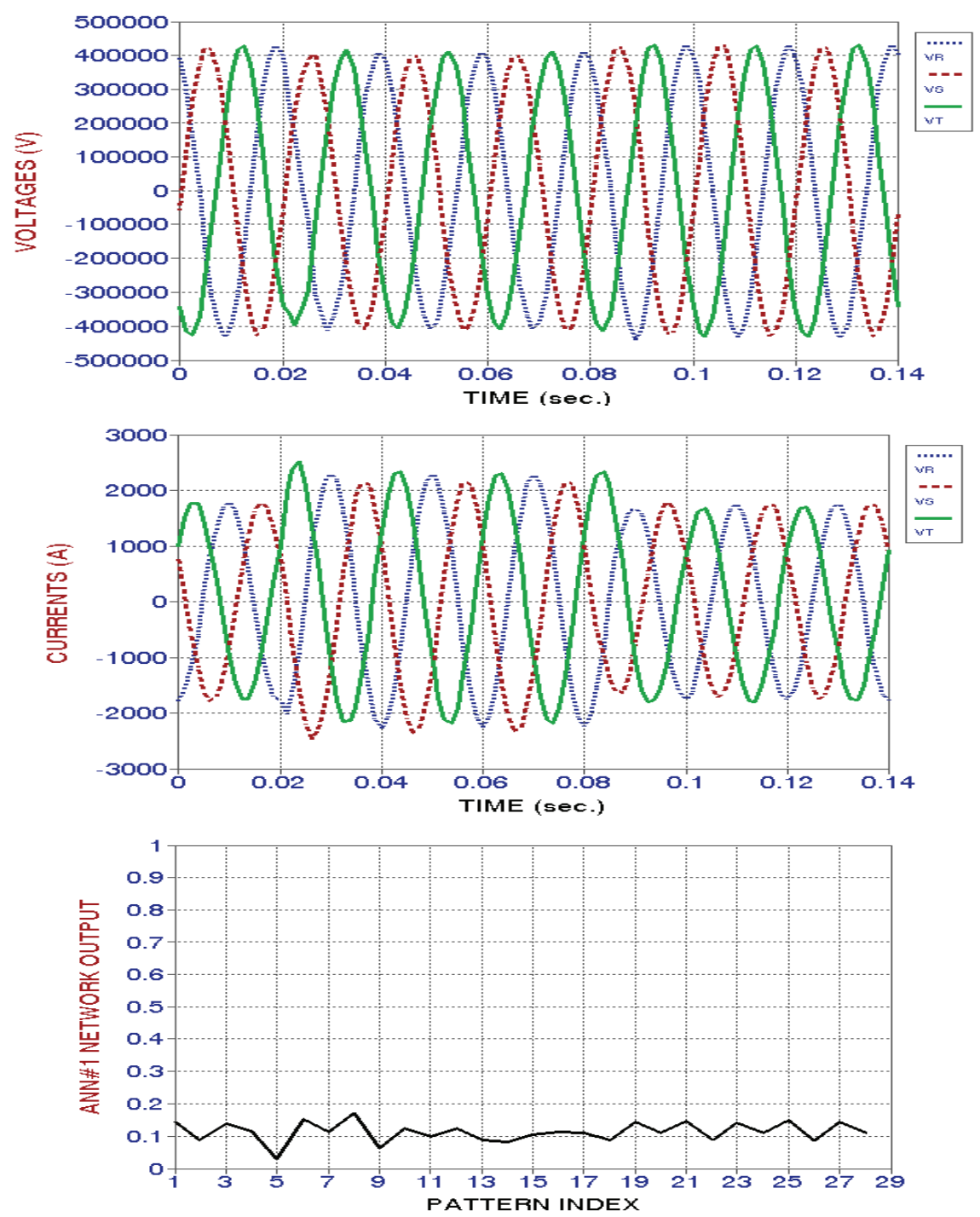

Fig. 15. Three phase voltages and currents at FDS and corresponding ANN outputs due to load switching at NH500.

\section{Conclusions}

A modular ANN based approach was proposed to design a FDS, as a main function of a high speed protective relaying scheme for the HV/EHV tapped TL's. The FDS main features are: identify the faulted 
zone, classify the type of fault and select the faulted phase. The proposed FDS consists of three hierarchical stages. The first stage is a preprocessing step, where the input measurements are processed via filtration, conversion, and normalization. The second stage consists of one ANN, which is responsible for detecting the faulted zone. In the third stage there are two zone diagnosis systems, each system consists of seven ANN's and is responsible for the classification of fault type as well as the selection of faulted phase in its zone. The investigation of the performance of the proposed FDS under various fault conditions leads to the following conclusions:

1. The proposed FDS reduces the cost and complexity of the relaying scheme for tapped lines.

2. The proposed architecture of the proposed FDS is constructed from 15 modular ANN's, with the advantage of assigning one task to each ANN, to enhance its learning ability and leading to a high quality performance.

3 . The adequate length of the used data window is $1 / 4$ cycle ( 4 samples of three phase voltages and currents at a sampling rate of 16 samples per cycle).

4. The time response of the proposed FDS is very fast due to its parallel structure. The expected time delay of the decision is about $1 / 3$ cycle.

5. The selected design for each modular ANN is suitable (24 input nodes, 24 hidden-neurons and one output-neuron).

6. The accuracy in the training phase was perfect $(100 \%)$, irrespective to fault location, fault type, and fault incipience time, while that of the testing phase is in the range (92\%-100\%).

7. The proposed FDS has proved high capability in classifying the transients produced from the capacitor and load switchings as normal cases.

8. The FDS can be used as a part of new generation of ultra-highspeed protective relaying schemes.

\section{References}

[1] Westinghouse Electric Corporation, Relay Investment Division, "Applied Protective Relaying", Coral Springs, Florida 33065(1982).

[2] Horowitz, S.H.G., Phadke, A., "Power System Relaying", RSPLTD, England (1992). 
[3] IEEE Working Group - Power System Relaying Committee, "Single Phase Tripping and Autoreclosing of Transmission Lines", IEEE Transactions on Power Delivery, 7, (1): January (1992).

[4] Sekine, Y., et al., "Fault Diagnosis of Power Systems", Proceedings of the IEEE, 80(5): 673-683, May (1992).

[5] CIGRE TF 38.06.06,"Artificial neural networks for power systems", Electra, 159: 77101, April (1995).

[6] El-Sharkawi, M.A. and Niebur, D., "A Tutorial Course on Artificial Neural Networks with Applications to Power Systems", IEEE copyright (1996).

[7] Aggarwal, R.K. and Johns, A.T., "Neural network based adaptive single-pole autoreclusure technique for ehv transmission systems", IEE Proc.C, 141(2): 155-160, March (1994).

[8] Zaman, M.R. and Rahman, M.A. "Experimental Testing of an Artificial Neural Network Based Protection of Power Transformer", IEEE Trans. on Power Delivery, 13, (2): 510517 (1998).

[9] Khamis, E.A., "Power Transmission System Fault Classification Using AI Technique", M.Sc. Thesis, Ain-Shams University, Sept. (2000).

[10] Mohamed, E.A., Abdelaziz, A.Y. and Mustafa, A.S., "A Neural Network Based Scheme for Fault Diagnosis of Power Transformers", EPSR Journal, 75: 29-39, July (2005).

[11] Dalstein, T., et al., "Neural network approach to fault classification for high speed protective relaying", IEEE Trans. Power Delivery, 10(2): 1002-1011, April (1995).

[12] Kezunovic, M. and Rikalo, I., "Detect and Classify Faults Using Neural Nets", IEEE Transactions on Computer Applications in Power, 9(4), October (1996).

[13] Song, Y.H. et al., "Comparison studies of five neural network based fault classifiers for complex transmission lines," EPSR, 43: 125-32(1997).

[14] Poeltl, A. and Frohlich, K., "Two new methods for very fast Fault type Detection by means of Parameter fitting and Artificial Neural Networks," IEEE Trans. on Power Delivery, vol. 14, no. 4, pp: 1269-1275, 1999.

[15] Lin, W.M., et al., "A fault classification method by RBF neural network with OLS learning procedure," IEEE Trans. on Power Delivery, 16(4): 473-477(2001).

[16] Yeo, S.M., et al., "A novel algorithm for fault classification in transmission lines using a combined adaptive network and fuzzy inference system," Electric Power and Energy Systems, 25: 747-758 (2003).

[17] Mohanty, R.N., et al., "Application of RBF neural network to fault classification and location in transmission lines," IEE Proc. Gen. Trans. Dist., 151(2): 201-212(2004).

[18] Omar, Y.A.S., 'Combined fuzzy-logic wavelet-based fault classification technique for power system relaying', IEEE Trans. Power Delivery, 19(2): 582-589(2004).

[19] Lahiri, U., et al., "Modular neural network-based directional relay for transmission line protection," IEEE Trans. on Power System, 20(4): 2154-2155(2005).

[20] Vasilic, S. and Kezunovic, M., "Fuzzy ART neural network algorithm for classifying the power system faults," IEEE Trans. Power Delv., 20(2), pt.2: 1306-14, Apr. (2005).

[21] Reddy, M.J. and Mohanta, D.K., "Adaptive-neuro-fuzzy inference system approach for transmission line fault classification and location incorporating effects of power swings", IEE Gener. Transm. Distrib., 2(2): 235-244 (2008).

[22] EMTP Developed Coordination Group, Electric Power Research Institute EMTP Rule Book, Version 2.1, Sections 6-10 (1993).

[23] Demuth, H. and Beal, M., Neural Network - Toolbox, using MATLAB The MathWorks Inc., Natick, MA, 2002 [Online]. Available: http://www.mathworks.com/. 


\section{Appendix A}

\section{Power System DATA}

The system data and operating conditions are given as follows:

\section{Generator data:}

\begin{tabular}{|c|c|c|c|c|c|c|c|c|}
\hline $\mathbf{X}_{\mathbf{d}}{ }^{\prime}$ & $\mathbf{X}_{\mathbf{d}}{ }^{\prime \prime}$ & $\mathbf{X}_{\mathbf{d}}$ & $\mathbf{X}_{\mathbf{q}}{ }^{\prime}$ & $\mathbf{X}_{\mathbf{q}}{ }^{\prime \prime}$ & $\mathbf{X}_{\mathbf{q}}$ & $\tau_{\mathbf{d}}{ }^{\prime}$ & $\mathbf{H}$ & $\mathbf{D}$ \\
\hline 0.4 & 0.37 & 1.2 & 0.36 & 0.16 & 0.79 & 2.8 & 6 & 0.18 \\
\hline
\end{tabular}

Where:

$\mathbf{X}_{\mathbf{d}}{ }^{\prime \prime}, \mathbf{X}_{\mathbf{d}}{ }^{\prime} \& \mathbf{X}_{\mathbf{d}}$ : d-axis subtransient, transient \& synchronous reactance's respectively;

$\mathbf{X}_{\mathbf{q}}{ }_{\mathbf{q}}{ }^{\prime \prime}, \mathbf{X}_{\mathbf{q}}{ }^{\prime} \& \mathbf{X}_{\mathbf{q}}$ : q-axis subtransient, transient \& synchronous reactance's respectively;

$\tau_{d}$ : d-axis transient time constant (sec); H: inertia time constant (sec); D: damping factor

2. Transformer Data: $206 \mathrm{MVA}, 15.75 \Delta / 500 \mathrm{Y} \mathrm{kV}, \mathrm{X}(\mathrm{LV})=0.006 \Omega, \mathrm{X}(\mathrm{HV})=1.67 \Omega$

\section{Transmission Lines Data:}

\begin{tabular}{|c|c|c|c|c|c|}
\hline$\#$ & From bus & To bus & $\mathbf{R}(\Omega)$ & $\mathbf{X}(\Omega)$ & $\mathbf{B}(\boldsymbol{\mu s})$ \\
\hline 1 & HD500 & NH500 & 2.56 & 34.80 & 460.2 \\
\hline 2 & NH500 & AS500 & 2.00 & 27.28 & 360.8 \\
\hline 3 & AS500 & SA500 & 1.75 & 23.75 & 308.1 \\
\hline 4 & SA500 & CA500 & 2.17 & 29.50 & 407.6 \\
\hline
\end{tabular}

\section{Operating Conditions:}

\begin{tabular}{|c|c|c|}
\hline Bus & $\mathbf{V ~ ( k V )}$ & Load (MVA) \\
\hline HD500 & $523.38 \angle 22.9$ & $10.32+\mathrm{j} 103.32$ \\
\hline NH500 & $507.70 \angle 11.8$ & $198.30+\mathrm{j} 132.17$ \\
\hline AS500 & $505.34 \angle 6.5$ & $85.12+\mathrm{j} 42.56$ \\
\hline SA500 & $496.70 \angle 3.2$ & $45.44+\mathrm{j} 40.89$ \\
\hline CA500 & $475.00 \angle 0$ & $157.43+\mathrm{j} 107.6$ \\
\hline
\end{tabular}


تصميم نظام تشخيص الأعطال لخطوط نقل القوى الكهربائية ذو ات التفريعة باستخدام الثبكات العصبية الاصطناعية

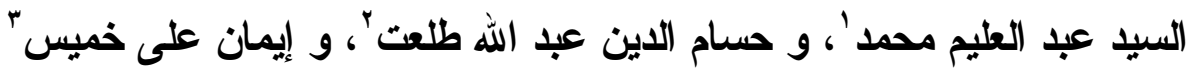

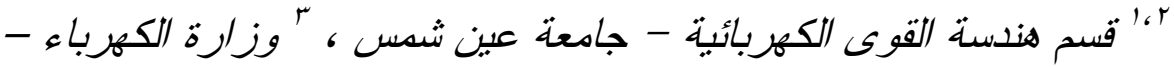

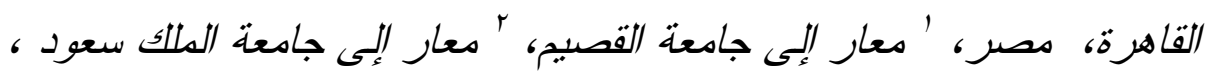

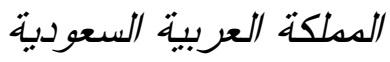

الستخلص. يتتاول هذا البحث تصميم نظام أو جهـاز لتهشخيص

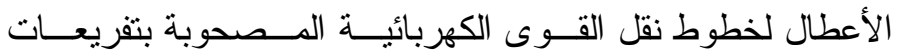
باستخدام الثبكات العصبية الاصطناعية، وروعي في التصميم قلة الإلة التكلفة الكلية، بالإضافة إلى بساطة التركيبات الداخلية لهذا الجهاز .

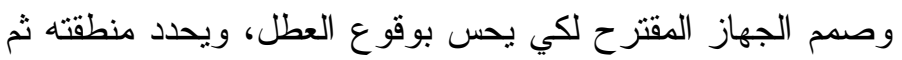

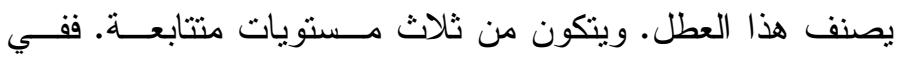

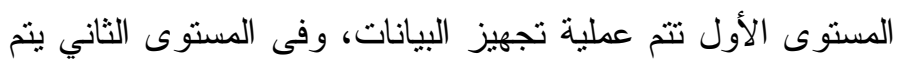

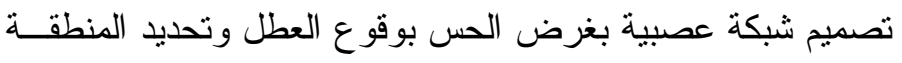

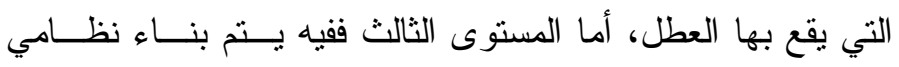

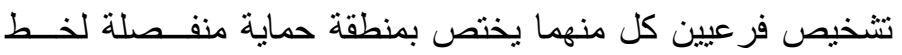

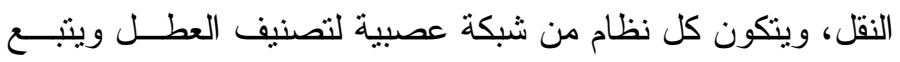

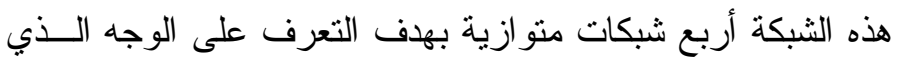

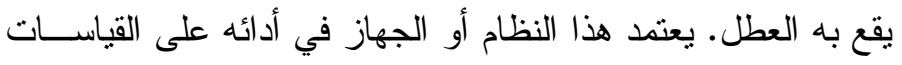

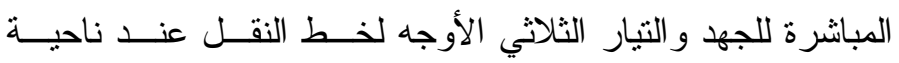

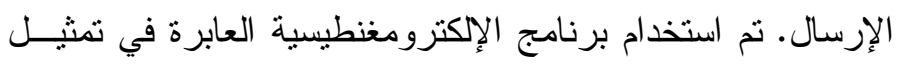

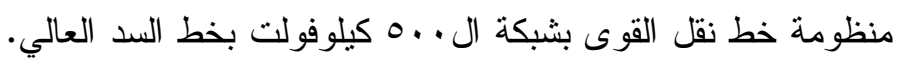

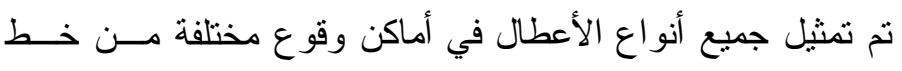

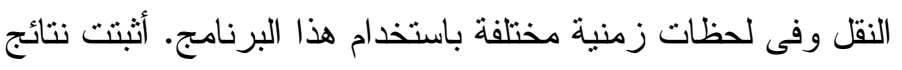
الدراسة دقة وكفاءة أداء جهاز النتخيص المقنت حـ 